\title{
Coexistence of giant aneurysm of sinus of Valsalva and coronary artery aneurysm associated with idiopathic hypereosinophilic syndrome
}

\author{
T Okinaka, N Isaka, T Nakano
}

\begin{abstract}
Aneurysms of the coronary sinuses of Valsalva and coronary artery aneurysms are uncommon cardiac anomalies, and cases in which these two uncommon lesions occur at the same time are extremely rare. A case of a woman with unstable angina who had a giant aneurysm of the left coronary sinus and multiple coronary artery aneurysms associated with an idiopathic hypereosinophilic syndrome is presented. Her sustained eosinophilia, elevated eosinophilic cationic protein concentration, and pathological findings of eosinophil infiltration of the aortic wall suggested the association of eosinophilia induced vascular injury as the cause of these aneurysms. This is the first such case to survive following surgical treatment. (Heart 2000;84:e7)
\end{abstract}

Keywords: aneurysm; arteritis; coronary sinus; eosinophilia

A 53 year old Japanese woman was admitted with recurrent episodes of anterior chest pain suggestive of unstable angina. She was known to have sustained eosinophilia without an apparent cause at the age of 47 . She had been temporarily treated with corticosteroids at which time she suffered from transient occlusions of the right central retinal artery. Over the previous five years she has been quite well without medication until one month before admission when she noticed anterior chest pain upon exertion. Physical examination on admission revealed the presence of a $2 / 6$ early diastolic murmur of aortic regurgitation. The ECG showed ST segment depressions from V2 to $\mathrm{V} 5$, and $\mathrm{T}$ wave inversions in I and aVL. The chest film showed enlargement of the left hilar area. The white cell count was $16400 / \mu$, with $55 \%$ eosinophils. Her erythrocyte sedimentation rate was $7 \mathrm{~mm} /$ hour. C-reactive protein and serum creatine phosphokinase were within normal range. The serum concentration of $\operatorname{IgE}$ was $1200 \mathrm{U} / \mathrm{ml}$, and the eosinophilic cationic protein (ECP) concentration was over $150 \mu \mathrm{g} / \mathrm{l}$ (normal $<15.7 \mu \mathrm{g} / \mathrm{l}$ ). Autoantibodies and antineutrophil cytoplasmic antibodies were negative. There was no evidence of lung or renal damage.

The root aortogram showed a coronary sinus of Valsalva aneurysm and enlargement of the right and non-coronary sinuses. Mild aortic regurgitation was also shown. The selective injection of contrast medium into the aneu- rysm revealed the size of the aneurysm to be $7.0 \times 6.0 \mathrm{~cm}$, and there was faint opacification of the left coronary artery. The right coronary artery showed diffuse segmental aneurysmal dilatation (fig 1). Left ventricular endomyocardial biopsy did not show endomyocardial fibrosis or inflammatory cell infiltration. Magnetic resonance angiography revealed that the thoracic and abdominal aorta as well as their branch origins were normal.

After administration of prednisone starting with $10 \mathrm{mg} / \mathrm{kg}$, the peripheral eosinophilic count was immediately normalised and the ECP concentration was decreased to $24 \mu \mathrm{g} / 1$. Two months after admission the operationwhich involved resection of the proximal aorta, placement of a composite graft containing a prosthetic aortic valve, and reimplantation of the coronary arteries into the graft-was successfully performed. Postoperative coronary
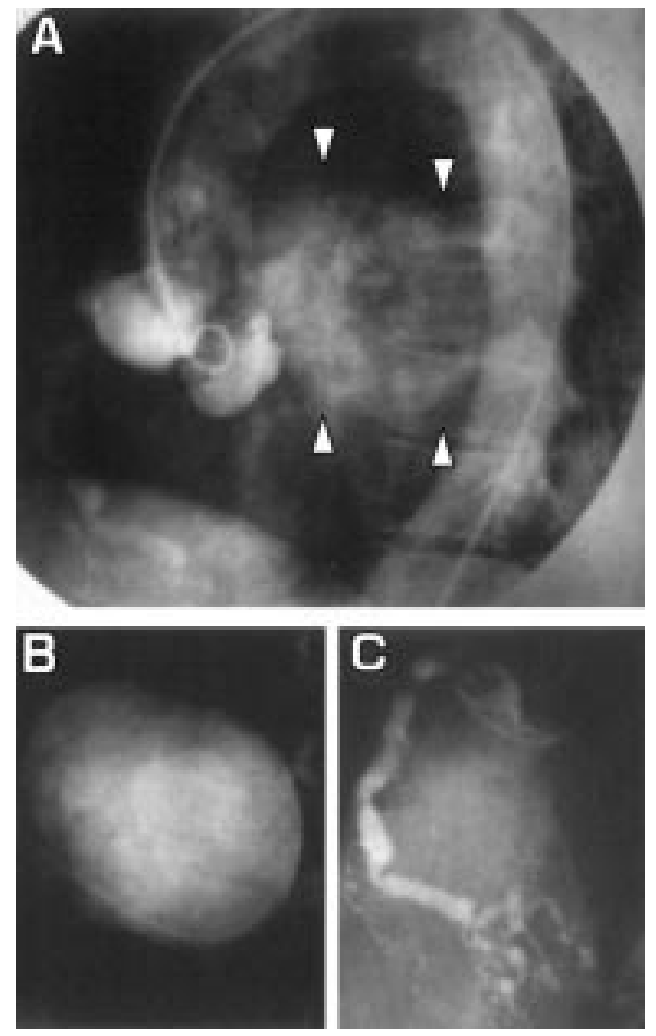

Figure 1 (A) Root aortogram showing a giant left sinus of Valsalva aneurysm (arrows), and enlarged right and non-coronary sinuses in left anterior oblique (LAO) projection. (B) A selective injection into the left coronary sinus aneurysm defining a large aneurysm connecting with the sinus in LAO projection. (C) Right coronary angiogram demonstrating multiple aneurysms in $L A O$ projection. 


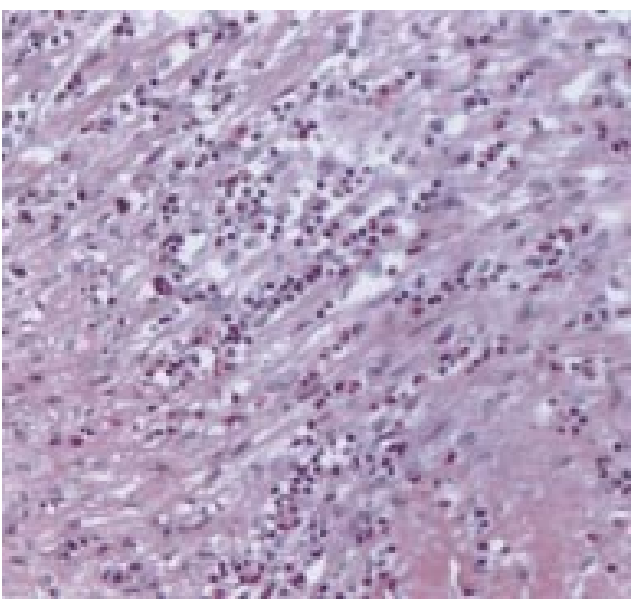

Figure 2 Histological section of the aorta with pronounced infiltration of eosinophils and mononuclear cells in its media (haematoxylin and eosin stain $\times 100$ ).

angiographies revealed segmentally dilated left ascending and circumflex arteries similar to the right coronary artery. Histological examination demonstrated the presence of pronounced infiltration of eosinophils and mononuclear cells along the intima and inner part of the media of the aortic wall extending to the aortic valves (fig 2). Mucinous degeneration was seen in the wall of the aneurysm but inflammatory cell infiltration was not prominent. The patient was free from angina and has continued to take prednisone, aspirin, and warfarin during a follow up period of three years.

\section{Discussion}

Aneurysms of the sinus of Valsalva are rare cardiac anomalies and acquired aortic sinus aneurysms are reported less frequently than congenital ones. ${ }^{1}$ In contrast to congenital coronary sinus aneurysms, over $99 \%$ of which affect the right or non-coronary sinuses, ${ }^{2}$ acquired coronary sinus aneurysms may arise from any of the sinuses. ${ }^{3}$ They are usually associated with acquired heart disease such as syphilis or endocarditis ${ }^{3}$ and commonly involve dilatation of all three coronary sinuses. ${ }^{2}$ In our patient, the aneurysm formation was of the left coronary sinus with coexistent dilatation of the right and non-coronary sinuses, suggesting that the condition was acquired.

Aneurysms of coronary arteries are also uncommon. Falsetti and Carroll reported an incidence of $1.5 \%$ of coronary aneurysms in an unselected catheterisation series of 732 patients. ${ }^{4}$ The majority of cases of coronary artery aneurysms in adults are acquired, usually as a result of atherosclerosis or secondary to inflammation caused by syphilis, infective endocarditis, or rheumatic carditis. ${ }^{5}$

Coexistence of a coronary sinus aneurysm and a coronary artery aneurysm is extremely rare and only three necropsied cases have been reported. They may have been either congenital or acquired. One case reported by Hudson mentioned the aneurysms were probably congenital. ${ }^{6}$ In the other two cases the aneurysms were considered to be acquired according to the pathological findings of inflammatory reactions of unknown causes. ${ }^{78}$ Our case is the first to survive following the diagnosis and undergoing treatment.

This patient met the diagnostic criteria for the idiopathic hypereosinophilic syndrome (HES) which requires sustained blood eosinophilia $>1500 / \mu \mathrm{l}$ persistent for longer than six months without an apparent aetiology or associated disease, and with evidence of organ involvement. ${ }^{9}$ In HES the cardiovascular involvement is the most severe and causes both morbidity and mortality. The typical cardiovascular manifestation of HES is endomyocardial damage and fibrosis with subsequent congestive heart failure as well as thrombosis and atrioventricular valve dysfunction. Although the endothelial damage has been thought to participate in the pathogenesis of the cardiovascular damage, vasculitis is not a prominent feature of this syndrome. ${ }^{10}$ In our patient raised ECP concentration may indicate eosinophil associated tissue injury, and histological evidence of eosinophil infiltration suggested eosinophil mediated vascular injury caused the coronary arteritis, coronary sinusitis, and subsequent development of the coronary sinus of Valsalva aneurysm and aortic regurgitation. This patient did not meet the classic criteria of either the Churg-Strauss syndrome, periarteritis nodosa, or temporal arteritis, all of which may be associated with eosinophilic vasculitis. Although vasculitis is not a prominent feature of HES, our case suggests that the coronary sinuses and coronary arteries may be the target tissue of this syndrome.

1 Goldberg N, Krasnow N. Sinus of Valsalva aneurysms. Clin Cardiol 1990;13:831-6.

2 Fushbein MC, Obma R, Roberts WC. Unruptured sunus of Valsalva aneurysm. Am f Cardiol 1975;35:918-22.

3 Jones AM, Langley FA. Aortic sinus aneurysms. Br Heart 7 1949;11:325-41.
Jones AM, Langley

4 Falsetti HL, Carroll RJ. Coronary artery aneurysm: a review of the literature with a report of 11 new cases. Chest 1976; 69: 630-6.

5 Virmani R, Robinowitz M, Atkinson JB, et al. Acquired coronary arterial aneurysms: an autopsy study of 52 patients. Human Pathol 1986;1 17:575-83.

6 Hudson REB. Aneurysm of the coronary artery. In: Cardiovascular pathology: London: Edward Arnold, 1970:398400.

7 Tangchai P, Buranavej C, Samranvej P. Aneurysms of right sinus of Valsalva and left coronary artery: an autopsy case report. F Med Assoc Thailand 1971;54:121-8.

8 Brewer JM, Bignold LP. Multiple coronary aneurysms with Brewer JM, Bignold LP. Multiple coronary aneurysms with
aortic sinus aneurysms. Arch Pathol Lab Med 1980;104: 592-4.

9 Weller PF, Bubley GJ. The idiopathic hypereosinophilic syndrome. Blood 1994;83:2759-79.

10 Fauci AS, Harley JB, Roberts WC, et al. The idiopathic hypereosinophilic syndrome. Ann Intern Med 1982;97:7892 